Supplementary information for

\title{
Inner shell excitation spectroscopy of biphenyl and substituted biphenyls: probing ring-ring delocalization
}

\author{
Jian Wang, Glyn Cooper, David Tulumello and Adam P. Hitchcock \\ Department of Chemistry, McMaster University, Hamilton, ON, Canada L8S 4M1
}

\section{$\underline{\text { Contents }}$}

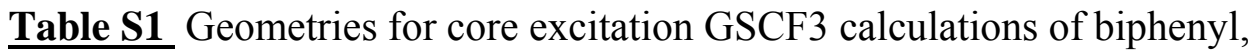
decafluorobiphenyl, benzene and hexafluorobenzene

Table S2: Selected eigenvalues, oscillator strengths, and orbital characters for computed core-excited states of $45^{\circ}$ twisted biphenyl and benzene

Table S3: Selected eigenvalues, oscillator strength, and orbital characters for computed $\mathrm{C} 1 \mathrm{~s}$ core-excited states of $60^{\circ}$ twisted decafluorobiphenyl and hexafluorobenzene

Table S4: Selected eigenvalues, oscillator strength, and orbital characters for computed F $1 \mathrm{~s}$ core-excited states of $60^{\circ}$ twisted decafluorobiphenyl and hexafluorobenzene

File: biphenyl-iseels-sup-info.doc

Last changed: 01-Oct-05 
Table S1: Geometries for core excitation GSCF3 calculations of biphenyl, decafluorobiphenyl, benzene and hexafluorobenzene

\begin{tabular}{|c|c|c|c|c|c|c|c|}
\hline \multicolumn{4}{|c|}{ Planar Biphenyl } & \multicolumn{4}{|c|}{ Planar Decafluorobiphenyl } \\
\hline \multirow{2}{*}{ Atom } & \multicolumn{3}{|c|}{ Coordinates $(\AA)$} & \multirow{2}{*}{ Atom } & \multicolumn{3}{|c|}{ Coordinates $(\AA)$} \\
\hline & $\mathbf{x}$ & $\mathbf{y}$ & $\mathbf{z}$ & & $\mathbf{X}$ & $\mathbf{y}$ & $\mathbf{z}$ \\
\hline $\mathrm{C} 1$ & 0.0000 & 0.0000 & 0.7509 & $\mathrm{C} 1$ & 0.0000 & 0.0000 & 0.7687 \\
\hline $\mathrm{C} 2$ & -1.1929 & 0.0000 & 1.5048 & $\mathrm{C} 2$ & -1.1792 & 0.0000 & 1.5633 \\
\hline $\mathrm{C} 3$ & -1.1966 & 0.0000 & 2.9072 & $\mathrm{C} 3$ & -1.1792 & 0.0000 & 2.9678 \\
\hline $\mathrm{C} 4$ & 0.0000 & 0.0000 & 3.6092 & $\mathrm{C} 4$ & 0.0000 & 0.0000 & 3.6717 \\
\hline $\mathrm{C} 5$ & 1.1966 & 0.0000 & 2.9072 & $\mathrm{C} 5$ & 1.1792 & 0.0000 & 2.9678 \\
\hline C6 & 1.1929 & 0.0000 & 1.5048 & C6 & 1.1792 & 0.0000 & 1.5633 \\
\hline C7 & 0.0000 & 0.0000 & -0.7509 & $\mathrm{C} 7$ & 0.0000 & 0.0000 & -0.7687 \\
\hline $\mathrm{C} 8$ & -1.1929 & 0.0000 & -1.5048 & $\mathrm{C} 8$ & -1.1792 & 0.0000 & -1.5633 \\
\hline C9 & -1.1966 & 0.0000 & -2.9072 & C9 & -1.1792 & 0.0000 & -2.9678 \\
\hline $\mathrm{C} 10$ & 0.0000 & 0.0000 & -3.6092 & $\mathrm{C} 10$ & 0.0000 & 0.0000 & -3.6717 \\
\hline $\mathrm{C} 11$ & 1.1966 & 0.0000 & -2.9072 & $\mathrm{C} 11$ & 1.1792 & 0.0000 & -2.9678 \\
\hline $\mathrm{C} 12$ & 1.1929 & 0.0000 & -1.5048 & $\mathrm{C} 12$ & 1.1792 & 0.0000 & -1.5633 \\
\hline $\mathrm{H} 1$ & -2.1661 & 0.0000 & 1.0245 & $\mathrm{~F} 1$ & -2.4020 & 0.0000 & 1.0282 \\
\hline $\mathrm{H} 2$ & -2.1417 & 0.0000 & 3.4442 & F2 & -2.3466 & 0.0000 & 3.6276 \\
\hline $\mathrm{H} 3$ & 0.0000 & 0.0000 & 4.6953 & F3 & 0.0000 & 0.0000 & 5.0106 \\
\hline H4 & 2.1417 & 0.0000 & 3.4442 & $\mathrm{~F} 4$ & 2.3466 & 0.0000 & 3.6276 \\
\hline H5 & 2.1661 & 0.0000 & 1.0245 & F5 & 2.4020 & 0.0000 & 1.0282 \\
\hline H6 & -2.1661 & 0.0000 & -1.0245 & F6 & -2.4020 & 0.0000 & -1.0282 \\
\hline $\mathrm{H} 7$ & -2.1417 & 0.0000 & -3.4442 & F7 & -2.3466 & 0.0000 & -3.6276 \\
\hline H8 & 0.0000 & 0.0000 & -4.6953 & F8 & 0.0000 & 0.0000 & -5.0106 \\
\hline H9 & 2.1417 & 0.0000 & -3.4442 & F9 & 2.3466 & 0.0000 & -3.6276 \\
\hline H10 & 2.1661 & 0.0000 & -1.0245 & F10 & 2.4020 & 0.0000 & -1.0282 \\
\hline \multicolumn{4}{|c|}{ Benzene } & \multicolumn{4}{|c|}{ Hexafluorobenzene } \\
\hline \multirow{2}{*}{ Atom } & \multicolumn{3}{|c|}{ Coordinates $(\AA)$} & \multirow{2}{*}{ Atom } & \multicolumn{3}{|c|}{ Coordinates ( $(\AA)$} \\
\hline & $\mathbf{x}$ & $\mathbf{y}$ & $\mathbf{z}$ & & $\mathbf{X}$ & $\mathbf{y}$ & $\mathbf{z}$ \\
\hline $\mathrm{C} 1$ & 0.0000 & 0.0000 & 1.3862 & $\mathrm{C} 1$ & 0.0000 & 0.0000 & 1.3862 \\
\hline $\mathrm{C} 2$ & 1.2005 & 0.0000 & 0.6931 & $\mathrm{C} 2$ & 1.2005 & 0.0000 & 0.6931 \\
\hline $\mathrm{C} 3$ & 1.2005 & 0.0000 & -0.6931 & $\mathrm{C} 3$ & 1.2005 & 0.0000 & -0.6931 \\
\hline $\mathrm{C} 4$ & 0.0000 & 0.0000 & -1.3862 & $\mathrm{C} 4$ & 0.0000 & 0.0000 & -1.3862 \\
\hline $\mathrm{C} 5$ & -1.2005 & 0.0000 & -0.6931 & $\mathrm{C} 5$ & -1.2005 & 0.0000 & -0.6931 \\
\hline C6 & -1.2005 & 0.0000 & 0.6931 & C6 & -1.2005 & 0.0000 & 0.6931 \\
\hline $\mathrm{H} 1$ & 0.0000 & 0.0000 & 2.4822 & F1 & 0.0000 & 0.0000 & 2.7692 \\
\hline $\mathrm{H} 2$ & 2.1496 & 0.0000 & 1.2411 & $\mathrm{~F} 2$ & 2.3982 & 0.0000 & 1.3846 \\
\hline $\mathrm{H} 3$ & 2.1496 & 0.0000 & -1.2411 & F3 & 2.3982 & 0.0000 & -1.3846 \\
\hline $\mathrm{H} 4$ & 0.0000 & 0.0000 & -2.4822 & F4 & 0.0000 & 0.0000 & -2.7692 \\
\hline H5 & -2.1496 & 0.0000 & -1.2411 & F5 & -2.3982 & 0.0000 & -1.3846 \\
\hline H6 & -2.1496 & 0.0000 & 1.2411 & F6 & -2.3982 & 0.0000 & 1.3846 \\
\hline
\end{tabular}


Table S2: Selected eigenvalues, oscillator strengths, and orbital characters for computed core-excited states of $45^{\circ}$ twisted biphenyl and benzene

\begin{tabular}{|c|c|c|c|c|c|c|}
\hline Site & IP & Character & Orbital & $\varepsilon(\mathrm{eV})$ & $f\left(10^{-2}\right)$ & $(R-\AA \AA)$ \\
\hline \multicolumn{7}{|c|}{ Biphenyl twisted $45^{\circ}$} \\
\hline \multirow[t]{8}{*}{$\mathrm{C} 1$} & 293.191 & $1 \pi^{*}\left(\mathrm{~b}_{1}\right)$ & 42 & -4.973 & 2.48 & 2.758 \\
\hline & & $1 \pi^{*}(\mathrm{a})$ & 45 & -3.411 & 0.00 & 2.767 \\
\hline & & $1 \pi^{*}\left(\mathrm{~b}_{3}\right)$ & 44 & -1.966 & 0.42 & 2.696 \\
\hline & & $1 \pi^{*}\left(\mathrm{~b}_{2}\right)$ & 43 & -1.672 & 0.00 & 2.824 \\
\hline & & $2 \pi^{*}\left(b_{1}\right)$ & 49 & 2.388 & 1.03 & 3.049 \\
\hline & & $\sigma^{*}(\mathrm{C}-\mathrm{C})$ (inter ring) & 46 & 2.521 & 0.80 & 2.447 \\
\hline & & $\sigma^{*}(\mathrm{C}-\mathrm{H})$ & 91 & 4.537 & 0.36 & 3.369 \\
\hline & & $\sigma^{*}(\mathrm{C}-\mathrm{H})$ & 72 & 5.319 & 0.28 & 3.150 \\
\hline \multirow[t]{8}{*}{$\mathrm{C} 2$} & 292.402 & $1 \pi^{*}\left(b_{1}\right)$ & 44 & -4.730 & 2.43 & 2.723 \\
\hline & & $1 \pi^{*}(\mathrm{a})$ & 45 & -3.503 & 0.12 & 2.833 \\
\hline & & $1 \pi^{*}\left(\mathrm{~b}_{2}\right)$ & 43 & -1.460 & 0.03 & 2.820 \\
\hline & & $1 \pi *\left(b_{3}\right)$ & 42 & -1.324 & 0.04 & 2.765 \\
\hline & & $\sigma^{*}(\mathrm{C}-\mathrm{H})$ (local) & 91 & 1.917 & 1.50 & 2.860 \\
\hline & & $2 \pi^{*}\left(b_{1}\right)$ & 49 & 2.665 & 0.85 & 2.932 \\
\hline & & $\sigma^{*}(\mathrm{C}-\mathrm{H})$ & 48 & 4.146 & 0.13 & 3.090 \\
\hline & & $\sigma^{*}(\mathrm{C}-\mathrm{H})$ & 54 & 4.812 & 0.44 & 3.188 \\
\hline \multirow[t]{9}{*}{$\mathrm{C} 3$} & 292.445 & $1 \pi^{*}\left(b_{1}\right)$ & 44 & -4.614 & 2.61 & 2.811 \\
\hline & & $1 \pi^{*}(\mathrm{a})$ & 45 & -3.619 & 0.03 & 2.724 \\
\hline & & $1 \pi^{*}\left(b_{3}\right)$ & 42 & -0.965 & 0.13 & 2.830 \\
\hline & & $1 \pi^{*}\left(b_{2}\right)$ & 43 & -0.936 & 0.01 & 2.818 \\
\hline & & $\sigma^{*}(\mathrm{C}-\mathrm{H})$ (local) & 53 & 1.559 & 1.40 & 4.035 \\
\hline & & $2 \pi^{*}\left(b_{1}\right)$ & 51 & 2.577 & 0.92 & 2.690 \\
\hline & & $\sigma^{*}(\mathrm{C}-\mathrm{H})$ & 48 & 4.394 & 0.15 & 3.598 \\
\hline & & $\sigma^{*}(\mathrm{C}-\mathrm{C})$ & 57 & 4.585 & 0.36 & 3.205 \\
\hline & & $\sigma^{*}(\mathrm{C}-\mathrm{C})$ & 58 & 5.963 & 0.37 & 3.235 \\
\hline \multirow[t]{7}{*}{$\mathrm{C} 4$} & 292.338 & $1 \pi^{*}\left(b_{1}\right)$ & 44 & -4.725 & 2.34 & 2.777 \\
\hline & & $1 \pi^{*}(\mathrm{a})$ & 45 & -3.209 & 0.00 & 2.820 \\
\hline & & $1 \pi^{*}\left(b_{3}\right)$ & 42 & -1.094 & 0.36 & 2.794 \\
\hline & & $1 \pi^{*}\left(b_{2}\right)$ & 43 & -0.965 & 0.00 & 2.814 \\
\hline & & $\sigma^{*}(\mathrm{C}-\mathrm{H})$ (local) & 53 & 1.651 & 1.41 & 4.541 \\
\hline & & $2 \pi^{*}\left(b_{1}\right)$ & 51 & 2.668 & 0.90 & 2.560 \\
\hline & & $\sigma^{*}(\mathrm{C}-\mathrm{H})$ & 48 & 4.475 & 0.13 & 3.331 \\
\hline \multicolumn{7}{|c|}{ Benzene } \\
\hline \multirow[t]{7}{*}{ C } & 292.536 & $1 \pi^{*} e_{2 u}$ & 22 & -4.812 & 2.72 & 1.725 \\
\hline & & $1 \pi^{*} \mathrm{e}_{2 \mathrm{u}}$ & 23 & -3.522 & 0.00 & 1.725 \\
\hline & & $\sigma^{*}(\mathrm{C}-\mathrm{H})($ local $)$ & 27 & 1.136 & 1.38 & 2.626 \\
\hline & & $2 \pi^{*} b_{2 g}$ & 25 & 2.497 & 1.03 & 1.697 \\
\hline & & $\sigma^{*}(\mathrm{C}-\mathrm{H})$ & 24 & 3.873 & 0.20 & 2.466 \\
\hline & & $\sigma^{*}(\mathrm{C}-\mathrm{C})$ & 29 & 4.470 & 0.27 & 2.350 \\
\hline & & $\sigma^{*}(\mathrm{C}-\mathrm{H})$ & 26 & 5.157 & 0.46 & 2.327 \\
\hline
\end{tabular}

* For $\pi^{*}$ orbitals, "local" means that the orbital is localized on one phenyl ring containing the $\mathrm{C} 1 \mathrm{~s}$ core hole; "delocalized" means that the orbital is distributed across two phenyl rings with usually more contribution in the ring with the core hole. For $\sigma^{*}$ orbitals, "local" means that the orbital is localized at the $\sigma$ bond(s) at the core hole site. 
Table S3: Selected eigenvalues, oscillator strength, and orbital characters for computed $\mathrm{C} 1 \mathrm{~s}$ core-excited states of $60^{\circ}$ twisted decafluorobiphenyl and hexafluorobenzene

\begin{tabular}{|c|c|c|c|c|c|c|}
\hline Site & IP & Character & Orbital & $\varepsilon(\mathrm{eV})$ & $f\left(10^{-2}\right)$ & $(R-\AA)$ \\
\hline \multicolumn{7}{|c|}{ Decafluorobiphenyl twisted $60^{\circ}$} \\
\hline \multirow[t]{9}{*}{$\mathrm{C} 1$} & 295.352 & $1 \pi^{*}\left(\mathrm{~b}_{1}\right)$ & 82 & -6.855 & 2.13 & 2.879 \\
\hline & & $1 \pi^{*}(\mathrm{a})$ & 85 & -5.093 & 0.00 & 2.837 \\
\hline & & $1 \pi^{*}\left(\mathrm{~b}_{3}\right)$ & 84 & -4.331 & 0.23 & 2.754 \\
\hline & & $1 \pi^{*}\left(\mathrm{~b}_{2}\right)$ & 83 & -3.412 & 0.00 & 2.889 \\
\hline & & $\sigma^{*}(\mathrm{C}-\mathrm{C})$ (inter ring) & 86 & -1.465 & 0.48 & 2.582 \\
\hline & & $2 \pi^{*}\left(b_{1}\right)$ & 89 & 0.333 & 0.58 & 3.151 \\
\hline & & $\sigma^{*}(\mathrm{C}-\mathrm{F})$ & 87 & 0.599 & 0.53 & 3.374 \\
\hline & & $\sigma^{*}(\mathrm{C}-\mathrm{F})$ & 88 & 1.176 & 0.72 & 2.927 \\
\hline & & $\sigma^{*}(\mathrm{C}-\mathrm{F})$ & 97 & 4.155 & 1.70 & 2.532 \\
\hline \multirow[t]{9}{*}{$\mathrm{C} 2$} & 296.822 & $1 \pi^{*}\left(\mathrm{~b}_{1}\right)$ & 84 & -6.845 & 2.52 & 2.839 \\
\hline & & $1 \pi^{*}(\mathrm{a})$ & 85 & -5.421 & 0.13 & 2.921 \\
\hline & & $1 \pi^{*}\left(\mathrm{~b}_{3}\right)$ & 82 & -3.781 & 0.12 & 2.799 \\
\hline & & $1 \pi^{*}\left(\mathrm{~b}_{2}\right)$ & 83 & -3.137 & 0.01 & 2.886 \\
\hline & & $\sigma^{*}(\mathrm{C}-\mathrm{F})$ (local) & 95 & -2.194 & 1.67 & 2.915 \\
\hline & & $2 \pi^{*}\left(b_{1}\right)$ & 92 & 0.325 & 0.81 & 3.063 \\
\hline & & $\sigma^{*}(\mathrm{C}-\mathrm{F})$ & 87 & 1.092 & 0.81 & 3.574 \\
\hline & & $\sigma^{*}(\mathrm{C}-\mathrm{F})$ & 97 & 4.284 & 1.22 & 3.108 \\
\hline & & $\sigma^{*}(\mathrm{C}-\mathrm{F})$ & 100 & 4.798 & 0.51 & 2.712 \\
\hline \multirow[t]{9}{*}{$\mathrm{C} 3$} & 296.514 & $1 \pi^{*}\left(\mathrm{~b}_{1}\right)$ & 84 & -6.535 & 2.18 & 2.881 \\
\hline & & $1 \pi^{*}(\mathrm{a})$ & 85 & -5.558 & 0.07 & 2.788 \\
\hline & & $1 \pi^{*}\left(\mathrm{~b}_{3}\right)$ & 82 & -3.355 & 0.16 & 2.890 \\
\hline & & $1 \pi^{*}\left(\mathrm{~b}_{2}\right)$ & 83 & -2.720 & 0.40 & 3.074 \\
\hline & & $\sigma^{*}(\mathrm{C}-\mathrm{F})$ (local) & 94 & -2.657 & 1.21 & 3.458 \\
\hline & & $2 \pi^{*}\left(b_{1}\right)$ & 92 & 0.111 & 0.97 & 2.837 \\
\hline & & $\sigma^{*}(\mathrm{C}-\mathrm{F})$ & 86 & 1.225 & 0.53 & 2.817 \\
\hline & & $\sigma^{*}(\mathrm{C}-\mathrm{F})$ & 90 & 4.068 & 0.68 & 3.262 \\
\hline & & $\sigma^{*}(\mathrm{C}-\mathrm{F})$ & 89 & 4.806 & 0.38 & 3.219 \\
\hline \multirow[t]{9}{*}{$\mathrm{C} 4$} & 296.823 & $1 \pi^{*}\left(\mathrm{~b}_{1}\right)$ & 84 & -6.866 & 2.49 & 2.853 \\
\hline & & $1 \pi^{*}(\mathrm{a})$ & 85 & -5.149 & 0.00 & 2.884 \\
\hline & & $1 \pi^{*}\left(b_{3}\right)$ & 82 & -3.383 & 0.17 & 2.832 \\
\hline & & $\sigma^{*}(\mathrm{C}-\mathrm{F})$ (local) & 112 & -2.785 & 1.70 & 3.966 \\
\hline & & $1 \pi^{*}\left(b_{2}\right)$ & 83 & -2.716 & 0.07 & 2.936 \\
\hline & & $2 \pi^{*}\left(b_{1}\right)$ & 94 & 0.197 & 1.01 & 2.651 \\
\hline & & $\sigma^{*}(\mathrm{C}-\mathrm{F})$ & 86 & 1.126 & 0.53 & 2.769 \\
\hline & & $\sigma^{*}(\mathrm{C}-\mathrm{F})$ & 88 & 2.663 & 0.36 & 3.535 \\
\hline & & $\sigma^{*}(\mathrm{C}-\mathrm{F})$ & 97 & 4.287 & 1.45 & 3.185 \\
\hline \multicolumn{7}{|c|}{ Hexafluorobenzene } \\
\hline \multirow[t]{7}{*}{$\mathrm{C}$} & 297.016 & $1 \pi^{*} \mathrm{e}_{2 \mathrm{u}}$ & 46 & -7.107 & 2.50 & 1.799 \\
\hline & & $1 \pi^{*} \mathrm{e}_{2 \mathrm{u}}$ & 47 & -5.724 & 0.00 & 1.795 \\
\hline & & $\sigma^{*}(\mathrm{C}-\mathrm{F})$ (local) & 50 & -4.008 & 1.69 & 2.274 \\
\hline & & $\sigma^{*}(\mathrm{C}-\mathrm{F})$ & 48 & -0.289 & 0.99 & 2.291 \\
\hline & & $2 \pi^{*} b_{2 g}$ & 52 & 0.017 & 0.95 & 1.739 \\
\hline & & $\sigma^{*}(\mathrm{C}-\mathrm{F})$ & 49 & 0.330 & 0.10 & 2.256 \\
\hline & & $\sigma^{*}(\mathrm{C}-\mathrm{C}-\mathrm{F})$ & 53 & 3.394 & 1.62 & 2.046 \\
\hline
\end{tabular}

* see footnote to table S2or meaning of "local", "delocalized" for $\pi^{*}$ orbitals and "local" for $\sigma^{*}$ orbitals. 
Table S4: Selected eigenvalues, oscillator strength, and orbital characters for computed F $1 \mathrm{~s}$ core-excited states of $60^{\circ}$ twisted decafluorobiphenyl and hexafluorobenzene

\begin{tabular}{|c|c|c|c|c|c|c|}
\hline Site & IP & Character & Orbital & $\varepsilon(\mathrm{eV})$ & $f\left(10^{-2}\right)$ & $(R-\AA)$ \\
\hline \multicolumn{7}{|c|}{ Decafluorobiphenyl twisted $60^{\circ}$} \\
\hline \multirow[t]{9}{*}{$\mathrm{F} 1$} & 694.186 & $1 \pi^{*}\left(\mathrm{~b}_{1}\right)$ & 82 & -5.758 & 0.12 & 2.759 \\
\hline & & $1 \pi^{*}(\mathrm{a})$ & 83 & -4.865 & 0.02 & 2.948 \\
\hline & & $1 \pi^{*}\left(b_{3}\right)$ & 85 & -3.510 & 0.01 & 2.823 \\
\hline & & $1 \pi^{*}\left(\mathrm{~b}_{2}\right)$ & 84 & -3.087 & 0.01 & 2.885 \\
\hline & & $\sigma^{*}(\mathrm{C}-\mathrm{F})$ (local) & 106 & -2.391 & 1.26 & 2.683 \\
\hline & & $2 \pi^{*}\left(b_{1}\right)$ & 89 & 1.058 & 0.03 & 3.055 \\
\hline & & $\sigma^{*}(\mathrm{C}-\mathrm{F})$ & 86 & 1.349 & 0.16 & 3.101 \\
\hline & & $\sigma^{*}(\mathrm{C}-\mathrm{F})$ & 92 & 3.569 & 0.04 & 3.185 \\
\hline & & $\sigma^{*}(\mathrm{C}-\mathrm{C}-\mathrm{F})$ & 94 & 5.578 & 0.05 & 2.569 \\
\hline \multirow[t]{9}{*}{ F2 } & 694.176 & $1 \pi^{*}\left(b_{1}\right)$ & 82 & -5.487 & 0.10 & 2.934 \\
\hline & & $1 \pi^{*}(\mathrm{a})$ & 84 & -4.974 & 0.02 & 2.723 \\
\hline & & $\sigma^{*}(\mathrm{C}-\mathrm{F})$ (local) & 85 & -3.231 & 0.71 & 3.210 \\
\hline & & $\sigma^{*}(\mathrm{C}-\mathrm{F})$ (local) & 113 & -2.706 & 0.63 & 3.272 \\
\hline & & $\sigma^{*}(\mathrm{C}-\mathrm{F})$ & 83 & -2.442 & 0.01 & 2.882 \\
\hline & & $2 \pi^{*}\left(b_{1}\right)$ & 89 & 0.908 & 0.04 & 2.857 \\
\hline & & $\sigma^{*}(\mathrm{C}-\mathrm{F})$ & 86 & 1.635 & 0.14 & 2.947 \\
\hline & & $\sigma^{*}(\mathrm{C}-\mathrm{F})$ & 93 & 3.904 & 0.05 & 3.278 \\
\hline & & $\sigma^{*}(\mathrm{C}-\mathrm{C}-\mathrm{F})$ & 97 & 5.781 & 0.05 & 3.020 \\
\hline \multirow[t]{9}{*}{ F3 } & 694.372 & $1 \pi^{*}\left(b_{1}\right)$ & 84 & -5.831 & 0.12 & 2.889 \\
\hline & & $1 \pi^{*}(\mathrm{a})$ & 85 & -4.555 & 0.00 & 2.801 \\
\hline & & $\sigma^{*}(\mathrm{C}-\mathrm{F})$ (local) & 106 & -3.108 & 1.38 & 4.002 \\
\hline & & $\sigma^{*}(\mathrm{C}-\mathrm{C})$ & 82 & -2.935 & 0.01 & 2.842 \\
\hline & & $\sigma^{*}(\mathrm{C}-\mathrm{F})$ & 83 & -2.354 & 0.00 & 2.877 \\
\hline & & $2 \pi^{*}\left(b_{1}\right)$ & 89 & 0.907 & 0.05 & 2.716 \\
\hline & & $\sigma^{*}(\mathrm{C}-\mathrm{F})$ & 86 & 1.464 & 0.10 & 2.765 \\
\hline & & $\sigma^{*}(\mathrm{C}-\mathrm{C}-\mathrm{F})$ & 93 & 4.072 & 0.08 & 3.332 \\
\hline & & $\sigma^{*}(\mathrm{C}-\mathrm{C}-\mathrm{F})$ & 97 & 5.616 & 0.11 & 3.012 \\
\hline \multicolumn{7}{|c|}{ Hexafluorobenzene } \\
\hline \multirow[t]{8}{*}{$\mathrm{F}$} & 694.155 & $1 \pi^{*} \mathrm{e}_{2 \mathrm{u}}$ & 46 & -5.995 & 0.11 & 1.779 \\
\hline & & $1 \pi^{*} \mathrm{e}_{2 \mathrm{u}}$ & 47 & -5.141 & 0.00 & 1.786 \\
\hline & & $\sigma^{*}(\mathrm{C}-\mathrm{F})$ (local) & 56 & -4.425 & 1.33 & 2.131 \\
\hline & & $\sigma^{*}(\mathrm{C}-\mathrm{F})$ & 48 & 0.068 & 0.21 & 2.289 \\
\hline & & $2 \pi^{*} b_{2 g}$ & 50 & 0.797 & 0.04 & 1.729 \\
\hline & & $\sigma^{*}(\mathrm{C}-\mathrm{F})$ & 49 & 0.988 & 0.03 & 2.265 \\
\hline & & $\sigma^{*}(\mathrm{C}-\mathrm{C}-\mathrm{F})$ & 51 & 2.359 & 0.12 & 2.169 \\
\hline & & $\sigma^{*}(\mathrm{C}-\mathrm{C}-\mathrm{F})$ & 53 & 4.652 & 0.06 & 2.036 \\
\hline
\end{tabular}

* see footnote to table S2or meaning of "local", "delocalized" for $\pi^{*}$ orbitals and "local" for $\sigma^{*}$ orbitals. 


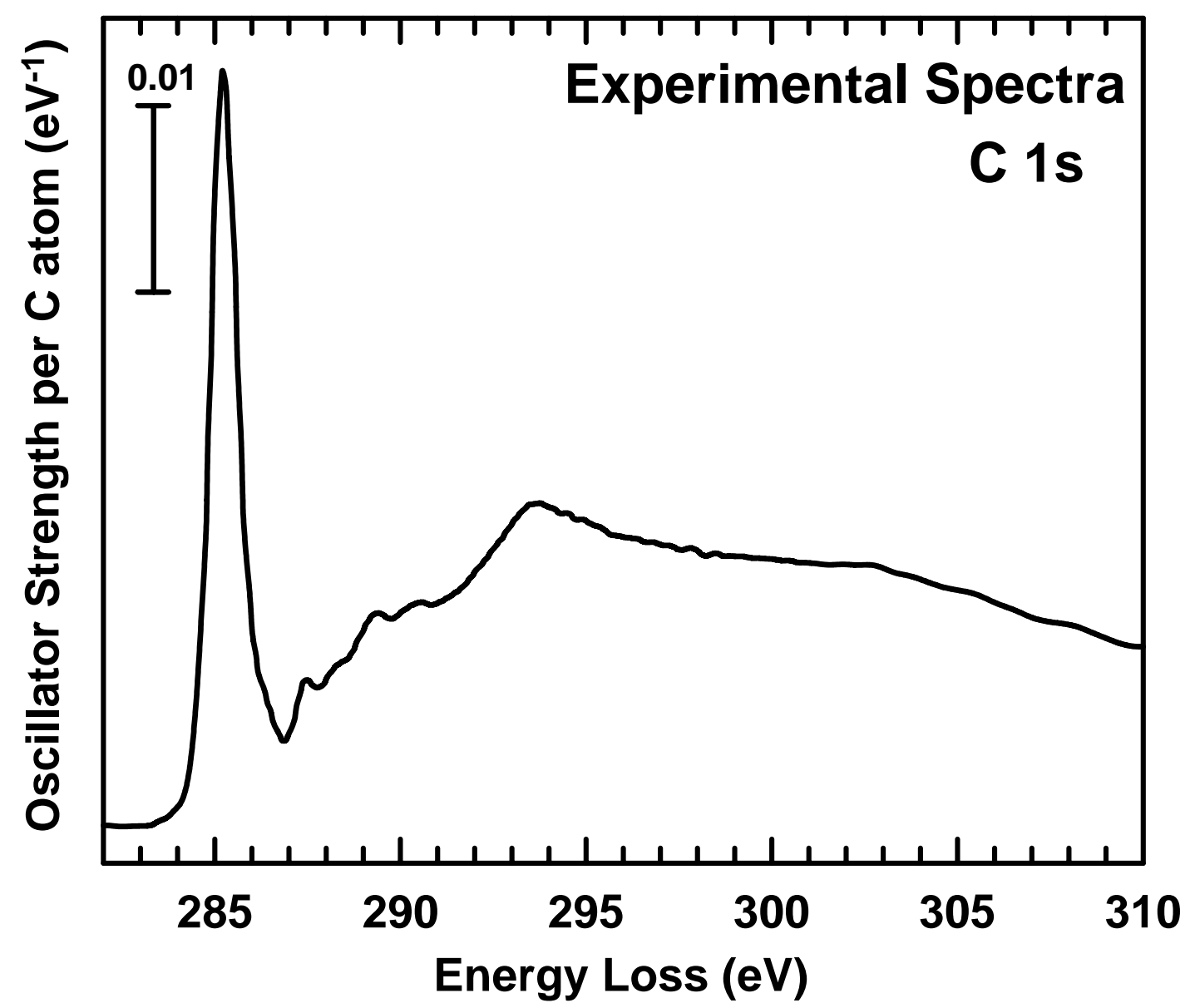

Fig S1 C 1s oscillator strength spectrum of gaseous 2,2'-bis(bromomethyl)-1,1'biphenyl. 